\title{
Research on Curricular Expanding Reading Education of Primary Chinese Study in the Web-based Classroom
}

\author{
Wang Manli \\ Wuchang Experimental Boarding Primary School, Wuhan, China \\ email: 437569695@qq.com
}

Keywords: web-based classroom environment; curricular expanding reading pedagogy; primary Chinese teaching

\begin{abstract}
As one of the methods to deepen the integration of information technology and classroom Chinese teaching, the curricular expanding reading education of primary Chinese study in the web-based classroom gives priority to primary school students in Chinese learning. This research, through its literature investigation on theories and practices of domestic expanding reading education of primary Chinese study, points out the current problems in the expanding reading education of primary Chinese study. Based on relevant teaching principles, this research announces the "Goal-focus" Chinese curricular expanding reading pedagogy under the cyber environment and also the construction of a curricular expanding reading system of theory and practice under web-based environment. The system is able to build a harmonious interactive platform for students and realize the integration of autonomous reading and collaborative reading, records and thinking, learning and evaluation.
\end{abstract}

\section{Introduction}

In primary Chinese education, teachers are ought to help students learn extracurricular knowledge and materials for deeper understanding of Chinese knowledge and better mastery of Chinese language.[1] As primary Chinese education plays a connecting role in the basic teaching system, it is necessary to help develop students' interest in reading and their ability of autonomous reading, improve the ability of logical analysis, and form independent reading style as well as thinking pattern, which can lay a solid foundation for Chinese learning in middle school and establish their own literary edifice. [2] Currently, most studies have placed great importance on text teaching and extracurricular extensive reading, but curricular expanding reading has not received much attention. [3] Under the circumstances, this article puts forward that curricular expanding reading should be the focus of studies on Chinese teaching. [4] As an urgent task, curricular expanding reading is an inevitable choice for the integration of information technology and Chinese courses, so it is determined as the starting point of this research.

\section{Problems in Curricular Expanding Reading Education of Primary Chinese Study in the Web-based Classroom}

\subsection{Lack of knowledge about the value of curricular expanding reading}

Many primary school Chinese teachers still use the exclusive vehicle of teaching in class - teaching materials. Instead of recommending excellent extracurricular reading materials for students, they focus on the texts in teaching materials and give students a lot of exercises based on the texts. Not only is it so boring and tedious, but also it leaves inadequate time and space for students to carry out expanding reading in class. [5] What such class presents to students are scattered knowledge fragments, therefore, students are incapable of grasping the soul and essence of the texts, and it is even more difficult to arouse students' lasting interest in reading. 


\subsection{Improper selection of curricular expanding reading materials}

Since primary Chinese teaching materials cover a wide range of topics and teaching resources under the cyber environment are rich and varied, a lot of teachers lack artistry in choosing and employing expanding resources, and their teaching is not able to revolve around teaching objectives and difficulties. Moreover, they include all the relevant materials in classroom teaching, which increases the knowledge capacity of class and also takes students' time for thinking and activity.

\subsection{Overuse of information technology}

A large amount of network resources are used in some primary Chinese classes and primary school students are dazzled by multimedia slides. Teaching has become the piling of materials, which cannot create sufficient opportunities for students to communicate and even hinders the realization of teaching objectives, leading to the Chinese classroom teaching of low efficiency.

\section{3. “Goal-focus” curricular expanding reading pedagogy}

"Goal-focus" curricular expanding reading pedagogy refers to a Chinese classroom teaching method that selects different types of curricular expanding reading resources and makes use of the information-based reading environment created by information technology to achieve "autonomous, cooperative and inquiry" learning basing on an overall analysis on teaching objectives of units or texts and the focus on current teaching objectives. [6] It aims to consolidate, deepen and transfer the current Chinese teaching objectives through curricular reading and communication under the information-based environment, so as to profoundly integrate information technology with Chinese courses. The pedagogy firmly grasps the core--teaching objectives, and develops reading around these objectives. Types of reading resources, reading content selection, reading tool design and reading quality evaluation all take teaching objectives as the focus. Unlike this pedagogy, partially expanding reading pedagogy only establishes the superficial correlation between reading resources and teaching objectives, which actually confuses learning contents and learning objectives. As a result, the contents are rich but the objectives may not be completed. After teaching objectives are clarified, reading resources can be divided into different types according to various teaching objectives. Under each type, a large number of reading resources from different aspects and with different degrees of difficulty can also be provided. These network resources, mainly composed of texts, pictures and a small amount of audios and videos, give students access to a wealth of reading resources within the same time length for learning in classrooms. In addition, the computer-based classroom online learning environment makes it possible for each student with a computer to freely choose reading contents and autonomously arrange reading paths in accordance with their own reading ability and preference. During autonomous reading, students can utilize a specially developed online reading tool to record their reading experience. For example, students can extract good words and sentences, comment on a wonderful passage and air opinions on the entire article. Furthermore, students are able to learn views of others on the same words, paragraphs and articles by setting up the partner circle for reading, so that they can understand different reading perspectives as well as reading feelings, and share their reading fruits. This is very important for teachers. The reading tool developed in this research is of great use for teachers. By looking at the background data, teachers can clearly know the overall reading time and the number of articles. They can also check the starting and ending time for reading, reading contents and numbers of each student, so as to have an idea of his or her reading quality.

\section{Construction of "Goal-focus” Curricular Expanding Reading Pedagogy in the Web-based Classroom}

As a teaching method to support the leap-forward teaching of Chinese in primary schools, "Goal-focus" curricular expanding reading pedagogy can be utilized in teaching of units and texts of different themes. Hence, in the implementation of teaching, teachers need to consider whether 
the pedagogy can take effect in all respects ranging from analysis of teaching objectives, analysis of learners' characteristics, implementation of teaching steps to selection and presentation of reading resources and application of online reading technical tools.

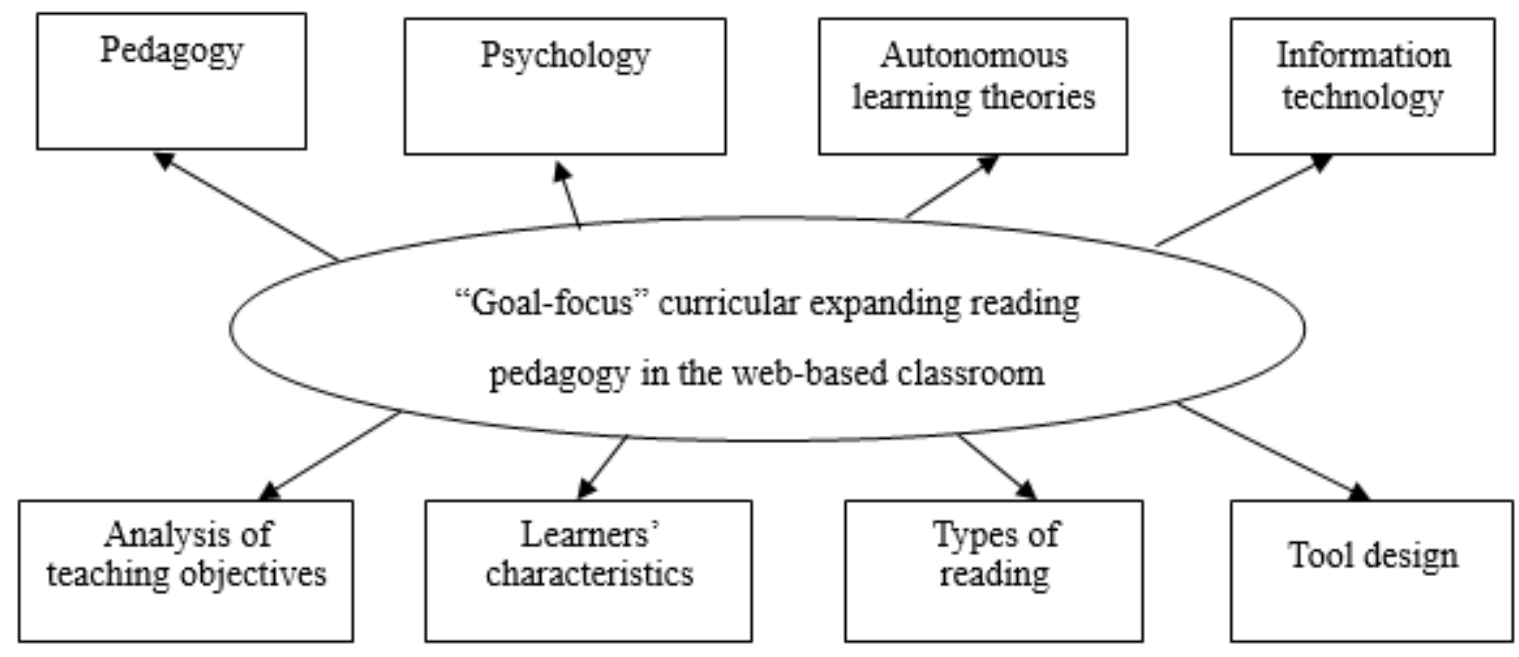

Figure 1 "Goal-focus” curricular expanding reading pedagogy in the web-based classroom

\subsection{Methods and techniques of teaching objectives analysis}

Teaching objectives are the cornerstone of implementing "Goal-focus" curricular expanding reading pedagogy. Under the guidance of macro teaching objectives for the second and third stages of Chinese curriculum standards, the analysis of units teaching objectives from a medium-level view is conducted and the analysis of units teaching objectives is decomposed into different texts. The micro teaching objectives of one text and even different teaching links can be achieved by taking advantage of multiple course hours. Therefore, a common method Analytic Hierarchy Process can be used to analyze the teaching objectives steps by steps from four different levels, successively that are, stage teaching objectives, unit teaching objectives, teaching objectives of course hour and teaching objectives of link.

\subsection{Analysis of learners' characteristics}

The purpose of teaching design is to effectively promote the study of learners, who represent the main body of learning activity. The characteristics of learners concerning cognition, emotion, intelligence and non-intelligence factors all have an impact on the information processing during learning. Thus analysis of learners' characteristics is a key factor to determine the success of teaching design. This research explores the teaching design of expanding reading from the perspective of deep integration of information technology and curriculum. So it mainly concerns learners’ information ability, reading ability, learning motivation and reading preference.

\subsection{Classification of expanding reading resources}

The leap-forward topic program adopts a relatively simple method for classifying articles of curricular expanding reading resources in lower grade classes. Basically, it is based on the principles that the first course hour aims to achieve literacy objective and the second course hour is targeted at cognition objective or emotion objective. In other words, there are two types of expanding reading articles for each text, namely literacy article and theme article, with about six to eight articles for each type. Although belonging to the same type, these six to eight articles differ in length, degree of difficulty and content, which can not only stimulate students' interest in reading and enrich their reading experience, but take account of learning diversity of students. Over the years, a large number of excellent expanding reading resources have been accumulated. However, 
as the teaching objectives of senior-grade classes become more detailed and in-depth, a more specific classification emerges, namely background article, theme article, article for accumulation, article for materials and sample article.

\subsection{IT tools to support and evaluate curricular expanding reading}

Digital resources and IT tools are indispensable to the establishment of an information-based learning environment. In the leap-forward test class under cyber environment, students have to read the online version of expanding reading resources on the teaching platform, which makes it even significant to utilize digital resources and IT tools. Since there is a lack of tools to support online reading, this research is dedicated to developing a tool that can support online reading.

a. Support for online reading

When reading online, students can discolor and underline words and sentences, and also can write annotations, just like they are reading on papers. By recording unique reading experience online, students' reading quality is sure to be improved and their records can be maintained on the Internet for a long time. What's more, the tool should make full use of the network environment to ensure that students can exchange reading experience with other peers and teachers in their classes or schools, and share reading fruits and wisdoms for a higher reading state.

b. Evaluation of online reading

The effect of implementing curricular expanding reading link is crucial to the leap-forward teaching under the web-based environment. The effect is determined by the quality of selected resources and the reading quality. The former should be completed by teachers and the latter by students. Therefore, to ensure the effective implementation of the link, the quality of expanding reading resources selected by teachers should be identified on the one hand, while the reading effectiveness of students are ought to be examined. For example, during the reading process, teachers can check whether students take it seriously, whether they read too quickly to save enough time for each material and whether the reading content is related to the types that teachers have specified. Moreover, teachers should pay special attention to inferior students, those with reading obstacles or poor self-management skills. They can also immediately respond to students who raise their hands and instruct students to adopt silent reading method and record by employing annotation and collection functions.

\section{Conclusion}

The core value of educational technology lies in finding and then solving problems in teaching practice. The effect and efficiency of teaching can be improved through the development of teaching system design, information-based teaching resources and tools, as well as innovative learning environment. This research, with its construction of teaching methods and design for teaching procedures, aims to solve the practical problems encountered by the leap-forward teaching program in the senior-grade primary Chinese education, and has completed the expected research goal. The advantages of "Goal-focus" curricular expanding reading pedagogy are that it only occupy classroom teaching time for reading and all reading resources are targeted at teaching objectives. In addition, the extinctive information-based reading environment is able to provide rich reading resources, elevate reading levels and evaluate reading quality of students who can share their reading fruits.

\section{References}

[1] Assessing the effects of gamification in the classroom: A longitudinal study on intrinsic motivation, social comparison, satisfaction, effort, and academic performance [J]. Michael D. Hanus, Jesse Fox. Computers \& Education. 2015

[2] Improving learning achievements, motivations and problem-solving skills through a peer assessment-based game development approach [J]. Gwo-Jen Hwang, Chun-Ming Hung, Nian-Shing 
Chen. Educational Technology Research and Development. 2014 (2)

[3] Interaction, Internet self-efficacy, and self-regulated learning as predictors of student satisfaction in online education courses [J]. Yu-Chun Kuo,Andrew E. Walker,Kerstin E.E. Schroder, Brian R. Belland. The Internet and Higher Education. 2014

[4] Using think - aloud protocols to investigate the translation process of foreign language learners and experienced translators (A). Barbosa, H. G., Neiva, A. M. S. Triangulating translation. 2003

[5] Structure mapping: A theoretical framework for analogy. Gentner D. Cognitive Science. 1983

[6] Writing from Sources: A Structure-Mapping Model. Nash J G, Schumacher G R, Calson B W. Journal of Education. 1993 